Instituto Internacional de Investigación y Desarrollo Tecnológico Educativo INDTEC, C.A.

DOI: https://doi.org/10.29394/Scientific.issn.2542-2987.2018.3.7.8.155-176

OAl-PMH: http://www.indteca.com/ojs/index.php/Revista Scientific/oai

\title{
Un Acercamiento a la Realidad: Iniciativas de Adopción del Cuadro de Mando Integral en Venezuela
}

(Proyecto en Desarrollo)

Autoras: Gloria Morella Torrealba Urdaneta Universidad Centroccidental "Lisandro Alvarado", UCLA tgloria@ucla.edu.ve Lara, Venezuela Yamileth Pastora Lucena López Universidad Centroccidental "Lisandro Alvarado", UCLA ylucena@ucla.edu.ve Lara, Venezuela

\section{Resumen}

Este trabajo constituye un estudio en desarrollo, cuyo objetivo es analizar experiencias de aplicación del Cuadro de Mando Integral (CMI) en Venezuela, con interés en los rasgos propios de dicha aplicación en el país. Esta investigación de campo, enmarcada en el paradigma cuantitativo, es descriptiva y adopta el método inductivo-deductivo. Se estudiarán diez organizaciones que emprendieron procesos de adopción del CMI en Venezuela, mediante la técnica de encuesta, con aplicación de cuestionarios, que se analizarán utilizando estadística descriptiva. Esta técnica se acompaña con la entrevista aplicada a tres expertos, que fue sometida al análisis de contenido. De estas entrevistas se derivan los resultados preliminares del estudio, entre los cuales figuran: predominio de grandes empresas y del sector manufacturero en la aplicación del CMl; despliegue incompleto del mismo en las organizaciones; y apego a la estructura original de dicha herramienta. Se destacan el apoyo de la alta gerencia y el uso de aplicaciones informáticas, entre otros factores críticos para la aplicación exitosa del CMI, mientras, la comprensión limitada del mismo y las dificultades para identificar objetivos estratégicos constituyen barreras a su implementación. Se concluye que en Venezuela la aplicación del CMI enfrenta limitaciones que involucran elementos básicos de su modelo teórico.

Palabras clave: gestión de empresas; gestión; control de gestión. 


\title{
An Approach to Reality: Adoption Initiatives of the Balanced Scorecard in Venezuela \\ (Project in Development)
}

\begin{abstract}
This work is still a study in development. Its purpose is to analyze the experiences of the Balanced Scorecard (BSC) application in Venezuela, focuses on the own features of its application in this country. This field research is from quantitative paradigm. It is descriptive and adopts the inductivedeductive method. Ten (10) organizations that undertook processes of BSC adoption will be studied in Venezuela through the survey technique, with the application of questionnaires, which will be analyzed with descriptive statistics. In addition, an interview which was submitted to content analysis will be made to three (3) experts. From these interviews were derived the preliminary results of this paper, which are: the predominance of large enterprises and the manufacturing sector in the implementation of the BSC, the incomplete deployment in organizations and the attachment to the original structure of this tool. The high management support and the use of computer applications are emphasized among other critical factors for the successful implementation of the BSC. The limited understanding of the BSC and the difficulties in identifying strategic objectives constitute barriers to its implementation. It is concluded that in Venezuela the BSC application faces limitations that involve basic elements of its theoretical model.
\end{abstract}

Keywords: business management; management; management control.

Date Received: 24-06-2017

Date Acceptance: 04-09-2017 


\section{Introducción}

El Cuadro de Mando Integral (CMI) ha sido catalogado en la literatura gerencial como una herramienta de amplia aceptación en el mundo organizacional y de indiscutible proyección en la investigación académica (Porporato y García, 2007, pág. 18). En el marco de este entusiasmo han emergido afirmaciones y evidencias empíricas que atribuyen al CMI un sitial sobresaliente, como herramienta de apoyo a la gerencia estratégica (Banchieri y Campa, 2012a, pág. 2; Urrea, Jiménez y Escobar, 2004, pág. 25).

Sin embargo, algunas investigaciones ponen en tela de juicio estas aseveraciones, al mostrar resultados según los cuales, dentro de los contextos analizados, la aplicación del Cuadro de Mando Integral abarca un porcentaje reducido de las empresas objeto de estudio (Banchieri y Campa, 2012b, pág. 14; Quesado, Aibar y Lima, 2012a, pág. 117), en contraposición a las afirmaciones que atribuyen a la referida herramienta un amplio arraigo en la realidad organizacional. Según sugieren Banchieri y Campa (2012c, pág. 14), esta disparidad pudiera explicarse, por la tipología de las empresas estudiadas, ya que los trabajos cuyos resultados muestran una vasta aplicación del CMI se enfocan en universos organizacionales constituidos por grandes empresas, originadas en países desarrollados.

A los planteamientos que refutan el extendido uso del CMI, se suman algunas posturas escépticas en torno a la validez de las hipótesis de este instrumento (Norreklit, 2000a, pág. 82). En el mismo orden de ideas, se ha proporcionado evidencia empírica que cuestiona el supuesto equilibrio existente entre los elementos financieros y no financieros que conforman la herramienta analizada (Lipe y Salteiro, 2000a, pág. 284). Adicionalmente, la literatura de gestión da cuenta de algunos estudios que aportan elementos para argumentar los tropiezos que enfrenta el CMI en su implantación (Waal y Counte, 2009a, pág. 377; Wagner y Kaufmann, 2004a, pp. 273-278).

A la luz de los contrastes y cuestionamientos expuestos, resulta 
razonable formular interrogantes respecto a la validez universal de las hipótesis del Cuadro de Mando Integral y la factibilidad de aplicarlo en toda índole de organizaciones, principalmente, si se considera el universo de las pequeñas y medianas empresas (Quesado et al., 2012b, pág. 93) y el contexto de los países que no pertenecen a la esfera de las economías desarrolladas. En esta línea analítica, se plantea el desafío de profundizar esfuerzos de investigación orientados a estudiar experiencias de aplicación del Cuadro de Mando Integral en ámbitos distintos a los de su contexto de origen, con miras a verificar la validez del modelo original para dichas realidades.

En atención a las inquietudes investigativas expuestas, este trabajo se propone estudiar iniciativas de aplicación del CMI en el contexto venezolano, con miras a despejar interrogantes del siguiente tenor: a). ¿cuál es el perfil de las organizaciones que han emprendido la implantación del CMI en Venezuela?; b). ¿cuál es el nivel de avance que han alcanzado las organizaciones venezolanas en la implementación de tal herramienta?; c). ¿qué adaptaciones han sido incorporadas al modelo del CMI en el contexto analizado?; d). ¿qué factores han favorecido o entorpecido la aplicación del Cuadro de Mando Integral en las organizaciones del país?; e). ¿cuál es el impacto de la aplicación del CMI en el logro de los objetivos estratégicos de las organizaciones venezolanas?

En función de las interrogantes de investigación formuladas, este trabajo se plantea como objetivo general analizar experiencias de aplicación del $\mathrm{CMI}$ en organizaciones del contexto venezolano, con el interés centrado en los rasgos y situaciones propias de dicha aplicación en nuestra realidad. Cabe destacar que la temática estudiada en este trabajo adolece de un gran vacío en Venezuela, ya que el grueso de las contribuciones que enfocan el Cuadro de Mando Integral constituye estudios proyectivos, que giran en torno al diseño de este tipo de herramienta para organizaciones específicas (Clemente y Ríos, 2016; Zambrano y Buyones, 2015). 
Resulta pertinente añadir que en la realidad venezolana actual cobra vigencia el papel de las herramientas de control de gestión, tales como el CMI, ante un entorno económico, social y político que incluye limitaciones sobre los márgenes de beneficio de las actividades lucrativas y regulaciones en torno a los consumos susceptibles de ser incluidos en las estructuras de costos, todo lo cual, exige gestionar variables críticas y optimizar procesos.

Este estudio constituye una investigación en desarrollo, razón por la cual, sólo se presentan resultados preliminares derivados de una de las técnicas de recolección de datos utilizadas, puntualmente, la entrevista a expertos, en la cual se abordan, de manera general, las interrogantes de investigación antes planteadas, excepto las consideraciones sobre el impacto de la aplicación del CMI en el logro de los objetivos estratégicos de las organizaciones usuarias del mismo.

El trabajo se inicia con unas breves notas sobre el modelo del CMI y el debate sobre la validez de sus premisas. A continuación, se presenta la metodología del estudio, seguida de los resultados preliminares del mismo. Como elemento final del trabajo, se formulan unas breves conclusiones.

\section{Bases Teóricas}

\subsection{Cuadro de Mando Integral.}

\subsubsection{Aspectos Generales.}

El Cuadro de Mando Integral fue introducido por Kaplan y Norton (1992a), constituye un modelo sobre cuya base es posible establecer un sistema de gestión de apoyo a la estrategia organizativa. Los creadores de esta herramienta argumentan que la misma "traduce la visión y estrategia de una empresa a un conjunto coherente de medidas de actuación" (Kaplan y Norton, 2000a, pág. 38). Por tanto, permite expresar la estrategia en términos de acciones y resultados, gracias a lo cual, puede ser comprendida por toda la organización (Terán, Sánchez y Ruiz, 2012, pág. 123). 
Según Kaplan y Norton (2000b, pág. 38) el CMI transforma la misión y la estrategia en objetivos e indicadores, agrupados en cuatro perspectivas: financiera, clientes, procesos internos y aprendizaje y crecimiento. Entre estas perspectivas se establecen relaciones causa-efecto que, igualmente, vinculan entre sí a los objetivos e indicadores contenidos dentro de ellas. El esquema del CMI permite equilibrar medidas financieras y no financieras; objetivos de corto y largo plazo; y medidas de resultado con inductores de actuación (Andel-Kader, Moufty y Laitinen, 2011, pág. 214).

La hipótesis central del Cuadro de Mando Integral plantea que la generación de rentabilidad para los accionistas se sostiene en la creación de valor para los clientes, que depende, a su vez, del desempeño en los procesos críticos para generar dicho valor, con base en las capacidades humanas, estructurales y tecnológicas de la organización. Esta dinámica se desarrolla a través de las relaciones causa-efecto entre las cuatro perspectivas del modelo, que se comentan en el apartado siguiente.

\subsubsection{Perspectivas del Cuadro de Mando Integral.}

La perspectiva financiera refleja los resultados finales del desempeño de la organización en términos financieros tradicionales y mide el éxito final en la ejecución de la estrategia. Contempla indicadores tales como, rentabilidad sobre inversión, crecimiento de ingresos y reducción de costos (Kaplan y Norton, 2004a, pág. 59). El aspecto central de esta perspectiva debe ser generar un crecimiento sostenible de valor a largo plazo a favor de los accionistas (pág. 69).

La perspectiva del cliente contiene la propuesta de creación de valor diferenciado y sostenible que permitirá atraer y retener al segmento del mercado seleccionado como cliente objetivo para la comercialización de los productos de la organización, lo que en opinión de Moro (2005, pág. 7), constituye el aspecto central de la estrategia de la misma. La proposición de 
valor para el cliente describe la combinación de "productos, precios, servicios, relación e imagen que la empresa ofrece a los clientes" (Kaplan y Norton, 2004b, pág. 70).

La perspectiva de procesos internos identifica los procesos críticos que permiten a la unidad de negocios entregar las propuestas de valor necesarias para atraer y retener a los clientes de los segmentos seleccionados y satisfacer las expectativas de rendimientos financieros de los accionistas (Malgioglio J., 2002, citado en Contreras y Pérez, 2009a, pág. 23).

Según Kaplan y Norton (2004c, pág. 61), la perspectiva del aprendizaje y crecimiento describe cómo se combinan los recursos humanos, la tecnología y el clima de la organización para dar soporte a los procesos internos de creación de valor. Los autores citados consideran a los tres elementos mencionados como activos intangibles y plantean, explícitamente, que la perspectiva del aprendizaje y crecimiento enfoca la significación de los activos en cuestión, como base de la estrategia organizativa.

\subsection{Cuadro de Mando Integral: del modelo teórico al mundo empírico}

Una de las vertientes que ha nutrido la literatura sobre el Cuadro de Mando Integral ha sido el debate sobre la validez de sus hipótesis y la factibilidad de aplicar las premisas del mismo, línea de trabajo que incluye entre sus derivaciones el estudio de los factores que favorecen o impiden el éxito de las experiencias de aplicación de este instrumento.

A continuación, se comentan algunos trabajos que han aportado elementos a la discusión generada alrededor de esta temática. Este contenido se divide en tres aspectos: validez de las premisas del CMI, factores que favorecen la implementación del mismo y barreras que obstaculizan su aplicación. 


\subsubsection{Validez de las premisas del CMI.}

La literatura que analiza la validez de las hipótesis planteadas por el CMI no refleja una postura unánime al respecto.

Desde el punto de vista de la crítica puramente teórica al modelo del CMI, destaca el análisis de Norreklit (2000b, pág. 82), quien argumenta que las relaciones planteadas en el marco de dicha herramienta no tienen carácter de causalidad, ya que no pueden ser objeto de verificación empírica de manera inobjetable. Agrega que estas relaciones son solamente lógicas.

Las evidencias ofrecidas por los estudios empíricos parecieran coincidir con los planteamientos de la autora aludida, ya que no aportan resultados concluyentes en torno a las relaciones causales del CMI. Los trabajos desarrollados por Davis y Albrigth (2004, pág. 145) y por Chen, Yamauchi, Kato, Nishimura, e Ito (2006, pág. 345), validan la relación entre el uso del CMI y el mejoramiento en el desempeño. En cambio, Ittner, Larcker y Randall (2003, pág. 735), no encontraron evidencia de asociación entre el uso de la referida herramienta y los resultados económicos. Por su parte, los hallazgos de Neely, Kennerley y Martínez (2004, pág. 771), revelan que, si bien la implementación del CMI tuvo un impacto positivo en las ventas y en las utilidades, el cambio no alcanzó a ser significativo.

En otro cuestionamiento a los supuestos del CMI, Lipe y Salterio (2000b, pág. 293), aportan elementos que ponen en tela de juicio el equilibrio entre el uso de las medidas financieras y no financieras. En una orientación similar, Banchieri y Campa (2012d, pág. 13) concluyen que las empresas dan mayor importancia a la perspectiva financiera del CMI,

\subsubsection{Factores que favorecen el éxito del CMI.}

Entre los estudios que analizan los factores favorables a la implementación del CMI, cabe mencionar el trabajo de Assiri, Zairi y Eid (2006a), el cual se basa en un modelo conformado por 27 factores críticos de 
éxito, con potencial de influir en la implementación del CMI. A partir de dicho modelo, el estudio desarrollado en 25 países permitió clasificar los factores de éxito del CMI en tres categorías: dominantes, principales y de soporte, de acuerdo con la valoración otorgada a los mismos por las organizaciones consultadas. En la primera categoría se incluyeron el compromiso de la gerencia, las características del equipo encargado del CMI y la identificación de las perspectivas. Entre los factores importantes figuran: claridad de la misión, visión, valores y estrategia; entrenamiento del personal para adaptarse a los cambios que conlleva el CMl; y automatización del mismo.

Finalmente, se clasificaron como factores de soporte los siguientes aspectos: integración del CMI como parte del sistema de gestión, sistemas de autoevaluación (por ejemplo, sistemas de calidad) vinculados al CMI y acabado final de las medidas y del modelo total del mismo.

\subsubsection{Factores que perjudican la implementación del CMI.}

En el marco de la temática que aborda los factores adversos a la viabilidad del modelo del $\mathrm{CMI}$, resulta pertinente mencionar el trabajo de Wagner y Kaufmann (2004b, págs. 273-278), quienes estudiaron un conjunto de grandes empresas, principalmente multinacionales, de origen alemán.

Entre los obstáculos que atentan contra el éxito en la implementación del CMI figuran: falta de compromiso; escaso apoyo de la alta gerencia; carencias en la formulación y comprensión de la visión y la estrategia; dificultades para identificar los objetivos estratégicos y las relaciones causaefecto; pérdida de sostenibilidad del proyecto por resistencia al cambio; dificultad para disponer de los datos sobre el desempeño; y ausencia de vinculación entre el CMI y el sistema de recompensas de la organización.

Por su parte, Waal y Counte (2009b, pág. 377), encuentran evidencia que señala como factores desfavorables al $\mathrm{CMI}$, la falta de cultura de medición del desempeño, el escaso compromiso de la gerencia y la carencia de 
sistemas de información para la medición de los resultados. De igual forma, Banchieri y Campa (2012e, pág. 11), identifican como limitantes de los procesos de implementación del CMI: tropiezos en la selección y confección de indicadores y carencias de información para el funcionamiento del CMI.

Los hallazgos previamente comentados son corroborados, en buena medida, por el trabajo de Quesado et al. (2012c, pág. 118), el cual destaca las dificultades al identificar indicadores, así como, al formular objetivos de los niveles operativos.

En el contexto venezolano solamente se encontró como antecedente relativo al estudio de las experiencias de aplicación del CMI, el trabajo de Contreras y Pérez (2009b), que se centra en la vinculación entre el Cuadro de Mando Integral y los sistemas de recompensas.

\section{Metodología}

\subsection{Tipo de Investigación.}

La investigación se enmarca en un paradigma apegado al enfoque cuantitativo, en el cual la rigurosidad metodológica para apreciar la realidad viene dada por el dato, o sea, se aplican instrumentos cuyos datos son codificados, tabulados y analizados para alcanzar las conclusiones. (Palella y Martins, 2010, pág. 40). En el marco de este enfoque, se adopta el método inductivo-deductivo, cuyo primer componente (inductivo) permite que los datos obtenidos de las muestras seleccionadas sirvan de base para formular planteamientos susceptibles de ser generalizados. Por su parte, los rasgos deductivos se aprecian en el análisis de los resultados, que son interpretados a luz de un marco teórico, a partir de lo cual, se generan nuevas conclusiones lógicas desde formulaciones generales.

La investigación es de carácter descriptivo, por cuanto se orienta a: caracterizar el ámbito organizacional de aplicación del CMI en Venezuela, así como, a identificar los rasgos que adopta esta herramienta en el marco de la 
realidad organizativa del país. Para Cerezal y Fiallo (2004), “...en este tipo de investigación se emplean, fundamentalmente, métodos empíricos...como son: la observación, las encuestas, las entrevistas, etc." (pág. 12).

\subsection{Variables.}

A partir del objetivo de investigación propuesto en este trabajo y los propósitos específicos que se derivan del mismo, se han identificado las variables de estudio que se indican en la Tabla 1, en la cual se detallan, igualmente, los elementos que describen dichas variables y los criterios o fuentes que sirven de base a tales elementos.

Tabla 1. Variables de Estudio.

\begin{tabular}{|c|c|}
\hline Obietivo & Variable \\
\hline $\begin{array}{l}\text { Caracterizar de organizaciones usuarias del } \\
\text { CMl en Venezuela }\end{array}$ & $\begin{array}{l}\text { - Sector de Actividad de acuerdo con el Instituto Nacional de Estadistica, } \\
\text { (2013) en su Clasificador Venezolano de Actividades Económicas } \\
\text { - Tamaño de las organizaciones (medido por número de trabajadores) } \\
\text { según el Instituto Venezolano de los Seguros Sociales (2017). } \\
\text { La clasificación de las organizaciones es la siguiente: } \\
\text { Pequeñas: Menos de } 50 \text { trabajadores } \\
\text { Medianas : Entre } 50 \text { y } 250 \text { trabajadores } \\
\text { Grandes: Más de } 250 \text { trabajadores }\end{array}$ \\
\hline $\begin{array}{l}\text { Analizar el alcance de la implementación del } \\
\mathrm{CMl} \text { en las organizaciones venezolanas }\end{array}$ & $\begin{array}{l}\text { - } \quad \text { Fase de avance en la implementación del CMI } \\
\text { - } \quad \text { Niveles de la organización que participan en la implementación del CMI }\end{array}$ \\
\hline $\begin{array}{l}\text { Describir la Estructura del } \mathrm{CMl} \text { aplicada en } \\
\text { las organizaciones venezolanas }\end{array}$ & $\begin{array}{l}\text { - } \text { Perspectivas adoptadas } \\
\text { - } \quad \text { Relaciones causa-efecto. }\end{array}$ \\
\hline $\begin{array}{l}\text { Identificar Factores favorables y } \\
\text { desfavorables a la implementacióndel CMl }\end{array}$ & $\begin{array}{l}\text { - Factores que favorecen la implementación del CMI } \\
\text { - Factores que obstaculizan la implementación del CMI }\end{array}$ \\
\hline $\begin{array}{l}\text { Evaluar Impacto del CMI en el cumplimiento } \\
\text { de objetivos organizacionales }\end{array}$ & $\begin{array}{l}\text { - Percepción de los entrevistados sobre el impacto del CMI en el } \\
\text { cumplimiento de objetivos organizacionales }\end{array}$ \\
\hline
\end{tabular}

Fuente: (Elaboración Propia).

\subsection{Recolección de Datos.}

A los efectos de la recolección de datos, se contempla el uso de dos (2) técnicas: la encuesta y la entrevista a expertos (semiestructurada). La encuesta se apoyará en el uso de dos (2) cuestionarios, cuya población objeto de estudio está representada por las organizaciones del contexto venezolano que hayan adoptado el CMI y lo utilicen en la actualidad. Puesto que no existe 
una base de datos de estas organizaciones, no es posible identificarlas ni cuantificarlas con precisión, por lo cual, se utilizará una muestra no probabilística intencional compuesta por diez (10) organizaciones. Según Carrera y Vásquez (2007, pág. 94), en este tipo de muestra, las unidades de la población son "escogidas de acuerdo con criterios previamente establecidos, seleccionando unidades tipo o representativas".

En este caso, los criterios adoptados para seleccionar la muestra son: que las organizaciones estén dispuestas a participar en el estudio; que sean identificadas a través de fuentes bibliográficas o referencias de consultores y/o estudiosos del tema en el país.

El primer cuestionario estará orientado a obtener información enfocada en los cinco (5) objetivos específicos de la investigación (ver Tabla 1) y será aplicado a los responsables del CMI de las organizaciones de la muestra. EI segundo instrumento está dirigido a los gerentes y supervisores de área de dichas entidades. En este cuestionario se excluyen los elementos relacionados con el perfil de las organizaciones consultadas y la estructura del CMI adoptada por las mismas, considerando que esta información de carácter general pudiera no estar al alcance del personal en cuestión. Los cuestionarios incluyen, principalmente, preguntas de respuestas cerradas, de selección simple y múltiple. Las preguntas relacionadas con los dos (2) últimos objetivos específicos adoptan la forma de escala tipo Likert.

La técnica de la entrevista a expertos se aplicó a tres (3) estudiosos de la temática relativa al $\mathrm{CMl}$, tanto en el campo académico, como en el de la consultoría. El propósito de la entrevista fue obtener información sobre la aplicación del CMI en Venezuela, vista desde la experiencia y criterio especializado de los entrevistados. Los aspectos enfocados en la entrevista responden a los cuatro primeros objetivos específicos. 


\subsection{Análisis de Datos.}

El análisis de los datos derivados de la aplicación de las encuestas estará basado en la estadística descriptiva, con la aplicación de análisis de frecuencias y medidas de tendencia central, específicamente, la moda, debido a que es compatible con la naturaleza nominal de las variables analizadas. En el caso de las entrevistas, fueron sometidas al análisis de contenidos producto de la interacción sujeto-objeto, con la finalidad de interpretar y comprender la información suministrada, a la luz de la teoría que sustenta el estudio.

\section{Resultados Preliminares}

Los resultados que se exponen seguidamente constituyen un avance de los hallazgos de la investigación, derivados de la aplicación de la técnica de entrevista semiestructurada. Los resultados que se muestran se refieren a los cuatro primeros objetivos específicos del trabajo, incluidos en la Tabla 1.

\subsection{Perfil de las organizaciones que han emprendido iniciativas de adopción del Cuadro de Mando Integral (CMI) en Venezuela.}

Como fue puntualizado anteriormente, el perfil de las organizaciones que han implementado el CMI en el contexto venezolano, se visualiza en dos dimensiones: el tamaño de la organización y el sector de actividad la misma.

En torno al primer elemento, los expertos consultados coinciden en señalar que las iniciativas de implantación del Cuadro de Mando Integral son adoptadas, mayormente, por grandes empresas, planteamiento que concuerda con los hallazgos de algunos estudios, cuya evidencia constata la existencia de una marcada relación entre la aplicación del CMl y la escala de negocios de la organización (Hoque y James, 2000, pág. 11).

Respecto al sector en el cual se enmarcan las organizaciones que han aplicado el $\mathrm{CMI}$ en el contexto venezolano, las respuestas ofrecidas por los entrevistados apuntan a un predominio del sector manufacturero, segmento 
que, según los consultados, concentra cerca de un $60 \%$ de las iniciativas de aplicación de la herramienta analizada. Según refiere uno de los expertos, este comportamiento se explica por la mayor urgencia del sector industrial en materia de aplicación de herramientas dirigidas a la gestión, ante la complejidad propia de sus procesos operativos y las dificultades planteadas a las industrias en el marco de la realidad actual del país. Este patrón sectorial es concordante con los resultados de Assiri et al. (2006b, pág. 940), cuyo estudio reporta la figuración dominante del sector manufacturero, como ámbito de implementación del CMI, con un porcentaje del 36,9\%.

\subsection{Alcance de la implementación del CMI en el contexto venezolano.}

Según manifiestan los consultados, la mayoría de las organizaciones que deciden adoptar el Cuadro de Mando Integral completan el diseño e implementación de esta herramienta. No obstante, el porcentaje que representan aquellas experiencias en las cuales el uso del el CMI se consolida y mantiene su continuidad como herramienta de gestión es, aproximadamente, un $40 \%$. Esta cifra coincide con el planteamiento de Waal y Counet (2009c, pág. 377), quienes estiman en un rango que varía entre $50 \%$ y $70 \%$ los casos fallidos de aplicación del CMI y otros sistemas de gestión.

Por otra parte, los expertos indican que el proceso de implementación del CMI no alcanza a todas las esferas de la organización y se extiende solamente hasta el eslabón de supervisores, lo que implica una incongruencia respecto al modelo conceptual del CMI, según el cual, el mismo debe ser desplegado en todos los niveles de la organización (Olve, Roy y Wetter, 2000, pp. 87-89). El hallazgo aludido es consistente con las evidencias empíricas reflejadas por Banchieri y Campa (2012f, pág. 12).

Según los consultados, los obstáculos que impiden el despliegue total del Cuadro de Mando Integral tienen que ver con la resistencia del personal de los niveles operativos y las dificultades cognitivas del mismo, lo que 
concuerda con los hallazgos de Wagner y Kaufmann (2004c, pág. 274).

\subsection{Estructura del $\mathrm{CMI}$ aplicada por las organizaciones venezolanas.}

De acuerdo con las afirmaciones de los entrevistados, de manera general, las organizaciones venezolanas que han adoptado el Cuadro de Mando Integral se apegan a la estructura conformada por las cuatro perspectivas del modelo original de Kaplan y Norton (1992b). Algunas variantes se introducen para el tratamiento de la responsabilidad social empresarial, con la inclusión de una quinta perspectiva, hallazgo que confirma los resultados ofrecidos por Costa, Jorquero y Méndez (2005, págs. 81-82).

\subsection{Factores que han favorecido o entorpecido la implementación y permanencia del CMI en organizaciones venezolanas.}

Entre los factores que los expertos consultados consideran determinantes para el éxito en la implementación del Cuadro de Mando Integral, figuran: compromiso de la gerencia, vinculación de esta herramienta con un sistema de compensaciones variables para el personal, existencia de elementos de planificación estratégica formalizados, uso de aplicaciones informáticas para apoyar el seguimiento de los indicadores, existencia de una persona o unidad responsable por el CMI y capacitación del personal en aspectos específicos de aplicación de este instrumento. Los factores de éxito identificados por los expertos entrevistados están incluidos en el modelo que sirve de base al estudio de Assiri et al. (2006c, págs. 940-949).

Por otra parte, los entrevistados reconocen como factores que conspiran contra la viabilidad del Cuadro de Mando Integral, los siguientes: falta de compromiso de la gerencia, escasa formación gerencial del personal; comprensión limitada del modelo; dificultades para identificar los objetivos estratégicos; falta de sistemas de soporte para los datos del desempeño. Estos hallazgos concuerdan con las evidencias proporcionadas por varios trabajos 
que abordan esta temática (Banchieri y Campa, 2012g, pág. 11; Waal y Counte, 2009d, pág. 377; Wagner y Kaufmann, 2004d, págs. 273-278).

\section{A Manera de Conclusiones}

Los resultados preliminares de la investigación, con base en las entrevistas a expertos, confirman, de manera general, los hallazgos derivados de investigaciones previas enmarcadas en diferentes contextos geográficos, lo que pudiera sugerir la existencia de patrones de comportamiento comunes que gravitan en torno a las iniciativas de implementación del CMI. En tal sentido, se ratifica que la inclinación a adoptar la herramienta analizada es más propia de las organizaciones de gran tamaño que de las pymes, ante lo cual, resulta pertinente profundizar en el estudio de los factores que inhiben la incursión de este último segmento empresarial en la aplicación del CMI.

En el contexto venezolano el modelo del CMI enfrenta limitaciones en la viabilidad de su implementación y permanencia, motivado a la presencia de algunos factores desfavorables que, en algunos casos, están asociados a elementos básicos del modelo teórico de esta herramienta, tales como dificultades en el despliegue del CMI a todos los niveles de la organización y tropiezos en la identificación de los objetivos estratégicos.

Además del impacto que ejercen los obstáculos ya mencionados en la implementación del CMI, se confirma el rol crítico de algunos factores como el compromiso de la alta gerencia, el entrenamiento del personal y el uso de aplicaciones informáticas, como elementos que apoyan el potencial de éxito en la implantación de la herramienta analizada. Se debe destacar que el último factor aludido puede actuar como barrera para la aplicación del CMI en Venezuela, considerando los elevados costos de la tecnología en el país.

Un panorama compatible con las especificaciones teóricas se manifiesta alrededor de la adopción de las perspectivas del CMI en el país, ya que, según refieren los consultados, predomina la estructura original 
conformada por las cuatro perspectivas básicas, lo que refleja que se han incorporado pocas adaptaciones de esta herramienta en el contexto nacional, en similitud con los hallazgos de otras realidades, que reflejan, igualmente, la aceptación del esquema general contenido en el modelo conceptual del CMI .

\section{Referencias}

Andel-Kader, M., Moufty, S., \& Laitinen, E. (2011). Balance scorecard development. A review of literature and directions for future research. En M. G. Andel-Kader (Ed.), Review of Management Accounting Research. New York, USA: Editorial Palgrave McMillan, págs. 214-239.

Assiri, A., Zairi, M. \& Eid, R. (2006a,b,c). How to profit from the Balance Scorecard. An implementation road map. Industrial Management \& Data Systems, 106 (7), págs. 937-952.

Banchieri, L. \& Campa, F. (2012a,b,c,d,e,f,g). El cuadro de mando integral: teoría o realidad. RIGC, 10 (20), junio-diciembre.

Carrera, L. \& Vásquez, M. (2007). Técnicas en el trabajo de investigación. Caracas, Venezuela: Editorial Panapo.

Cerezal, J. \& Fiallo, J. (2004). Cómo investigar en pedagogía. La Habana. Cuba: Editorial Pueblo y Educación.

Chen, X, Yamauchi, K., Kato, K., Nishimura, A. \& Ito, K. (2006). Using the Balanced Scorecard to measure the chinese and japanese hospital performance. International Journal of Health Care Quality Assurance, 19 (4), págs. 339-350.

Clemente, C. \& Ríos, K. (2016). Desarrollo de un sistema de Cuadro de Mando Integral para la empresa Rialca, Servicios Industriales y Petroleros, C.A. Municipio Baruta, año 2015. Trabajo de Grado para optar al título de Licenciado en Administración. Caracas, Venezuela: Universidad Nueva Esparta. 
Contreras, J. \& Pérez, S. (2009a,b). Cuadro de Mando Integral y la compensación variable ejecutiva en las empresas del área Metropolitana de Caracas. Trabajo de grado para optar al título de Licenciado en Relaciones Industriales. Caracas, Venezuela: Universidad Católica Andrés Bello.

Costa, H., Jorquero, G. \& Méndez, L. (2005). Cuadro de Mando Integral: Un Estudio Exploratorio de la Experiencia Chilena. Trabajo para Optar al Título Ingeniero en Información y Control de Gestión. Universidad de Chile, Santiago.

Davis, S. \& Albright, T. (2004). An investigation of the effects of balance scorecard on financial performance. Management Accounting Research, 15 (2), junio, págs. 135-153.

Hoque, Z. \& James, W. (2000). Linking balanced scorecard measures to size and market factors: Impact on organizational Performance. Journal of Management Accounting Research, 12, págs. 1-17.

Ittner, C., Larcker, D. \& Randall, T. (2003). Performance implications of strategic performance measurement in financial services firms. Accounting, Organization and Society, 28, págs. 715-741.

Kaplan, R. \& Norton. D. (1992a,b). The Balance Scorecard: measures that drive performance. Harvard Business Review, january-february, págs. 71-79.

Kaplan, R. \& Norton, D. (2000a,b). El Cuadro de Mando Integral. Segunda Edición. Barcelona. España: Gestión 2000.

Kaplan, R. \& Norton, D. (2004a,b,c). Mapas Estratégicos. Barcelona. España: Gestión 2000.

Lipe, M. \& Salteiro, S. (2000a,b). The balance scorecard: judgmental effects of common and unique performance measures. The Accounting Review, 75 (3), págs. 283-298.

Malgioglio, J. (2002). Distintos enfoques del capital intelectual. Jornadas 
de Investigación de la Facultad de Ciencias Económicas y Estadísticas. Colombia: Universidad de Rosario.

Moro, M. (2005). Capital intelectual y cuadro de mando integral: la fuerza de la unión. XIII Congreso AECA, Oviedo.

Neely, A., Kennerley, M. \& Martinez, V. (2004). Does the balance scorecard work? an empirical investigation. Performance Measurement and Management Public and Private, págs. 763-773.

Norreklit, H. (2000a,b). The balance on balance scorecard: A Critical Analysis of some of its Assumptions. Management Accounting Research, 28, págs. 591-619.

Olve, N., Roy, J. \& Wetter, M. (2000). Implantando y gestionando el Cuadro de Mando Integral. Barcelona, España: Gestión 2000.

Palella, S. \& Martins, F. (2010). Metodología de la Investigación Cuantitativa. 2da edición. Caracas, Venezuela: Fondo Editorial de la Universidad Pedagógica Experimental Libertador.

Porporato, M. \& García N. (2007). Análisis de las limitaciones del cuadro de mando integral: revisión de literatura e implicaciones para la práctica. Revista Iberoamericana de Contabilidad de Gestión, 5 (9), págs. 13-32.

Quesado, P., Aibar, B. \& Lima, L. (2012a,b,c). El Cuadro de Mando Integral como herramienta de gestión estratégica en PYMES portuguesas. Revista Internacional de la Pequeña y Mediana Empresa, 1 (4), págs. 90-128.

Terán, O. E., Sánchez, I. \& Ruíz M. E. (2012). Cuadro de Mando Integral (CMI) como herramienta para identificar el comportamiento del capital humano. Omnia, 18 (1), págs. 121-134.

Urrea, J., Jiménez, A. \& Escobar, N. (2004). Aplicación del Cuadro de Mando Integral en proyectos de empresas sociales. Revista Universidad EAFIT, 40 (133), págs. 22-34. 
Waal, de A. A. \& Counet, H. (2009a,b,c,d). Lessons learned from performance management systems implementation. Industrial Journal of Productivity and Performance Management, 58 (4), págs. 367-390.

Wagner, S. \& Kaufmann, L. (2004a,b,c,d). Overcoming the main barriers in initiating and using purchasing-BSCs. Journal of Purchasing \& Supply Management, 10, págs. 269-281.

Zambrano, Y. \& Bullones. H. (2015). Propuesta de un sistema de indicadores de gestión basado en el Cuadro de Mando Integral para la Superintendencia de Estimación de Costos de la Refinería EI Palito de Petróleos de Venezuela S.A. Trabajo Especial de Grado para optar al título de Magister Scientiarum en Gerencia de la Construcción. Valencia, Venezuela: Universidad de Carabobo. 


\section{Gloria Morella Torrealba Urdaneta}

e-mail: tgloria@ucla.edu.ve

Nacida en Venezuela. Es Licenciada en Contaduría

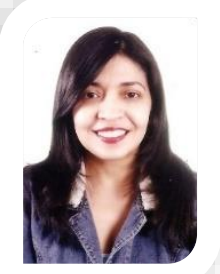
Pública, egresada de la Universidad Centroccidental "Lisandro Alvarado", en el año 1986. Posteriormente, se tituló Magister en Planificación Administrativa, Mención Gerencia Pública, en la misma institución, en 1996. Obtuvo el título de Doctora en Gestión y Administración de Empresas, por la Universidad de Valladolid (España), en 2008. Es profesora titular del Decanato de Ciencias y Tecnología de la Universidad Centroccidental "Lisandro Alvarado". Es autora de varios artículos de investigación en la línea de control de gestión y Economía Social. Es Coordinadora de la Unidad de Investigación en Ciencias Sociales del mismo decanato. 


\section{Yamileth Pastora Lucena López \\ e-mail: ylucena@ucla.edu.ve}

Natural de Barquisimeto, Estado Lara, Venezuela. En

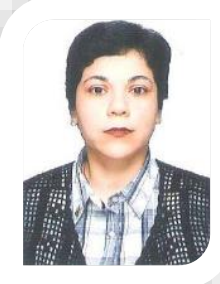
1997 egresa como Licenciada en Ciencia Política. Para el 2002 se convierte en Magister en Gerencia Empresarial (UFT). En 2013 obtiene el título de Doctora en Gerencia Avanzada. Actualmente cursa estudios de Especialización E-learning (UCLA) y Maestría en Comunicación Corporativa (UFT) (ambos en trabajo de grado). Profesora con categoría de Asociado en la Universidad Nacional Abierta, Centro Local Lara y de Agregado en la Universidad Centroccidental "Lisandro Alvarado" (UCLA). Posee experiencia como Profesora de Postgrado en las asignaturas relacionadas con las áreas de metodología y gerencia empresarial en la UFT, UNEFA y UNA.

El contenido de este manuscrito se difunde bajo una Licencia de Creative Commons Reconocimiento- 